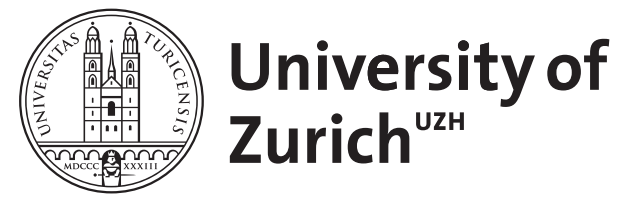
Archive

University of Zurich

University Library

Strickhofstrasse 39

CH-8057 Zurich

www.zora.uzh.ch

Year: 2020

Trans*Spezies - Intersektionale Hybridwesen in der afrofuturistischen Kunst von Juliana Huxtable

\author{
Jirka, Nadine
}

DOI: https://doi.org/10.1515/fs-2020-0020

Posted at the Zurich Open Repository and Archive, University of Zurich ZORA URL: https://doi.org/10.5167/uzh-192306

Journal Article

Published Version

Originally published at:

Jirka, Nadine (2020). Trans*Spezies - Intersektionale Hybridwesen in der afrofuturistischen Kunst von Juliana Huxtable. Feministische Studien, 38(2):201-218.

DOI: https://doi.org/10.1515/fs-2020-0020 


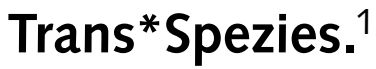

\section{Intersektionale Hybridwesen in der afrofuturistischen Kunst von Juliana Huxtable}

Die jüngste Einzelausstellung der US-amerikanischen Künstlerin, Autorin und DJane Juliana Huxtable mit dem Titel Interfertility Industrial Complex: Snatch the Calf Back ${ }^{2}$ gleicht einem grotesken Fiebertraum: Von den Wänden der Galerie blicken mehrere hypersexualisierte Mensch/TierHybridwesen, deren obszöne, weiblich markierte Körper in kompromittierenden Posen inszeniert sind. Fingierte, sensationalistische Boulevardschlagzeilen wie »SEX SLAVERY AND `ANIMAL HUSBANDRY - THE BEASTIALITY BEHIND YOUR BEEF" platzieren die absurden Figuren in einen satirischen Diskurs um die ethische Vertretbarkeit von industrieller Nutztierhaltung, während die Toilettenkabinen in der Mitte des Raumes die Betrachtenden - vermeintlich im Widerspruch dazu - diskret zu sexuellen Handlungen an den unterwürfigen Tierwesen einzuladen scheinen (Abb. 1). Für die Bilder von menschlich-tierischen Avataren hybridisiert Huxtable ihren eigenen Körper und bettet ihn ein in eine Verweisstruktur teilweise widersprüchlicher Signifikanten in Form von Symbolen und Parolen aus der Protestkultur, ornamentalen Strukturen und medialen Schocktaktiken, die sie nutzt, um den Themenkomplex Mensch / Tier auf vielschichtige Weise zu erkunden: Über die Schlagzeile »FURRIES GONE TOO FAR" rekurriert Huxtable beispielsweise auf eine on- und offline gelebte Subkultur, die ihre (oftmals erotisch fixierten) Identifikationen mit anthropomorphisierten Tieren ausleben, indem sie ihre "Fursona« - eine selbstgewählte anthropomorphe Identität - zumeist mittels Kostümierung performen (Reysen et al., 2020). Sie evozieren auf verstö-

1 Mein Dank gilt den anonymen Gutachter_innen, deren wertvolle kritischen Rückmeldungen meine Argumentation maßgeblich beeinflusst und vorangetrieben haben. Der Asterisk soll hervorheben, dass trans ${ }^{\star}$ hier auch als Methode und nicht nur als Identitätskategorie gemeint ist. Trans (ohne Asterisk) bezeichnet eine Bewegung quer durch und darüber hinaus. Die aus den transgender studies stammende Konzeptualisierung von trans` bezeichnet eine transversale Methodologie, die danach fragt, wie sich gendering zu Prozessen der Rassifizierung, Dehumanisierung, Speziesierung und Animalisierung verhält. (Vgl. Klumbyte 2018).

2 Galerie Reena Spaulings Fine Art, New York, 26. September - 27. Oktober 2019. 
rende Weise Fragen etwa danach, was wäre, wenn sich Menschen mittels genetischer Manipulation tatsächlich zu tierischen Hybridwesen umgestalten könnten, welche Rechte ihnen dann zukämen und ob ihnen der Status `Mensch aberkannt werden würde. Die spöttische Antwort aus Huxtables Parallelwelt lautet: »PLEASE STOP - FEMINISTS PLEAD WITH COW IDENTIFIED YOUTUBER TO LEAVE INTERSECTIONALITY ALONE«. Huxtable übersetzt somit reale Debatten um transfeminine Körper und Identitäten in eine utopisch / dystopische Fantasie, in welcher der Selbstidentifikation potentiell keine Grenzen gesetzt sind. ${ }^{3}$ Sie forciert auf diese Weise nicht nur eine Auseinandersetzung mit den Widerständen, auf die eine radikale Liberalisierung von Selbstidentifikation stoßen würde, sondern veranschaulicht auch - so wird dieser Beitrag zeigen - inwiefern Animalisierung und Unterdrückung von minoritären Subjekten miteinander in Verbindung stehen.

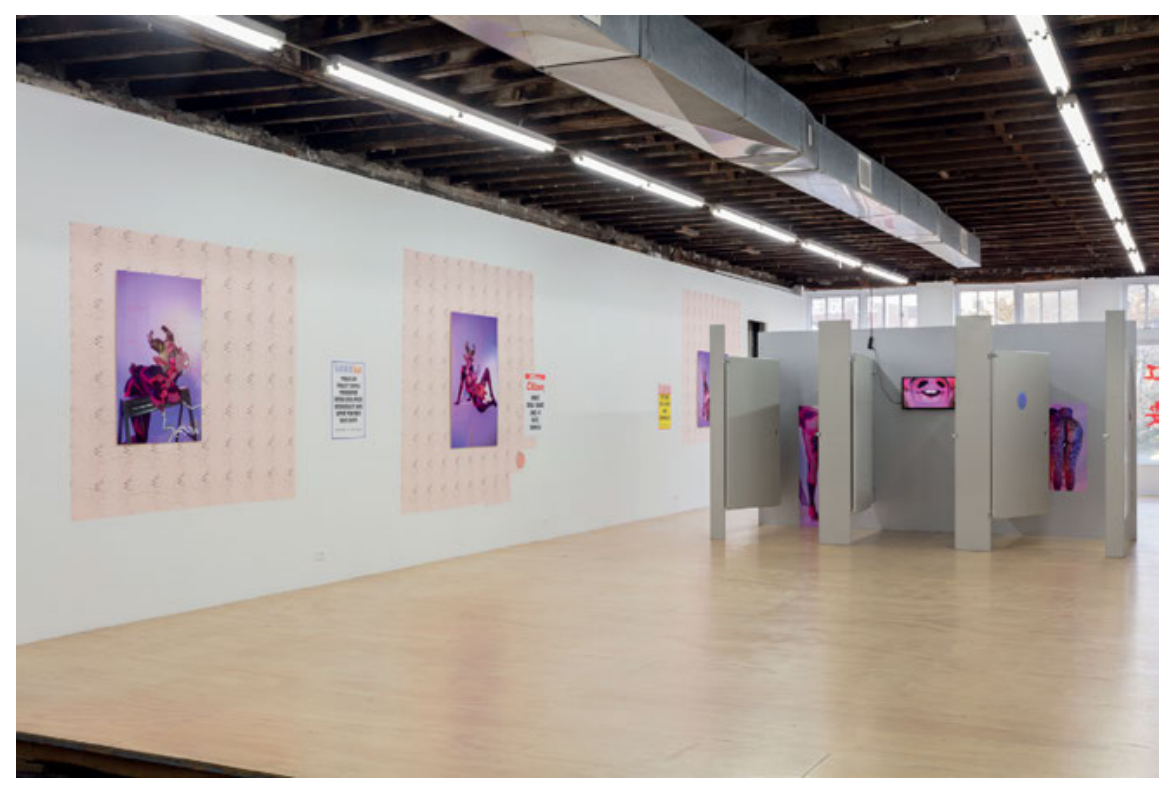

Abb. 1: Ausstellungsansicht, Reena Spaulings Fine Art New York, Einzelausstellung Juliana Huxtable "INTERFERTILITY INDUSTRIAL COMPLEX: SNATCH THE CALF BACK« 2019, Courtesy of the artist and Reena Spaulings Fine Art, NY/LA, Ausstellungsfoto (C) Joerg Lohse.

3 Die vorangegangene Schlagzeile aus der Ausstellung scheint eine ironisch überspitzte Anspielung auf Äußerungen sogenannter TERF (trans-exclusionary radical feminists) zu sein, in deren Augen Trans ${ }^{\star}$ Frauen von feministischen Bewegungen und prinzipiell von der Kategorie "Frau« ausgeschlossen sein sollten. 
Durch ein close reading von Werken aus zwei im Jahr 2019 eröffneten Ausstellungen Juliana Huxtables will ich mit diesem Beitrag die dargestellten Hybridwesen als intersektionale Figuren erkennbar machen, durch deren spezifische Körperinszenierungen Überschneidungen der Kategorien von Geschlecht und race (und in der Folge auch Klasse) deutlich gemacht werden. In der Inszenierung des transgeschlechtlichen und rassifizierten Körpers der Schwarzen Künstlerin überlagern sich mehrere Bedeutungsebenen, die Huxtable visuell mit dem Komplex der Beziehungen von Mensch und Tier in Verbindung bringt. Das Setting der industriellen Nutztierhaltung, das Huxtable in ihren Bildern aufruft, legt nahe, dass es sich bei der Diskriminierung und (sexuellen) Ausbeutung von Schwarzen (Trans ${ }^{\star}$ ) Frauen und der Ausbeutung von Tieren durch Menschen um vergleichbare Systeme der Unterdrückung handelt, die beide auf Dehumanisierung beruhen. $^{4}$

Die Trans`spezies-Wesen Huxtables lese ich im Folgenden als posthumane Denkfiguren, in denen sich gelebte Realitäten rassifizierter, vergeschlechtlichter und animalisierter minoritärer Subjekte mit futuristischen Visionen nichtmenschlicher Existenzformen verbinden, um eine Kritik an dominanten rassistischen, heteronormativen und speziesistischen Diskursen und Politiken zu formulieren. Ich diskutiere damit Huxtables Afrofuturismus als queerfeministische Strategie der Erkenntnisproduktion, mittels welcher neue Denk- und Erzählmodi generiert werden. ${ }^{5}$

\section{Afrofuturismus: Identifikation mit dem reptilen Alien}

Juliana Huxtables Einzelausstellung Zoosexuality an der Art Basel Hong Kong $2019^{6}$ zeigte sechs auf Leinen gedruckte fotografische Selbstporträts, die eine Zukunft imaginieren, in welcher die Grenzen zwischen Mensch und Tier durch Möglichkeiten gentechnologischer Manipulation durchlässig geworden sind (vgl. Abb. 2). Diese Beschreibung könnte eben so gut dem Plot eines Science-Fiction-Narrativs entstammen, das über eine

4 Der Vergleich von verschiedenen Systemen der Oppression bedeutet nicht eine Gleichsetzung menschlicher Erfahrungen mit denjenigen von Tieren.

5 Den Anstoß zu diesen Überlegungen gab der Ausstellungskatalog zu der letztjährigen Ausstellung "Producing Futures - An Exhibition on Post-Cyber-Feminisms« im Migros Museum für Gegenwartskunst, Zürich, in welcher Huxtable mit einem Werk vertreten war (vgl. Munder 2019, 87). Allerdings wird im Katalog nicht auf die Strömung des Afrofuturismus referiert.

6 Huxtable wurde an der Art Basel Hong Kong 2019 vertreten von der Galerie Project Native Informant in London. 
Zukunft spekuliert, in der technologischer Fortschritt unbegrenzte Möglichkeiten der körperlichen Transformation eröffnet. Huxtable zielt jedoch auf eine Vision, die mehr mit der Gegenwart und der Vergangenheit zu tun hat als mit der Zukunft. Ihre futuristischen Mensch / Tier-Mischwesen dienen der Künstlerin, wie sie in einem Interview bestätigt, als hyperbolische Tropen, um zeitgenössische Debatten um Identität und Repräsentation außerhalb eines statischen frameworks zu adressieren:

I find a lot of the questions around representation and identity to be really literal, discussed in terms that can only operate under set, didactic rubrics. And I feel like if you're going to talk about gender or the intersection of gender, race, and representation - at least insofar as art, or my art, has to answer this - it's more interesting to just go trans-species. (Huxtable in Busta, 2019)

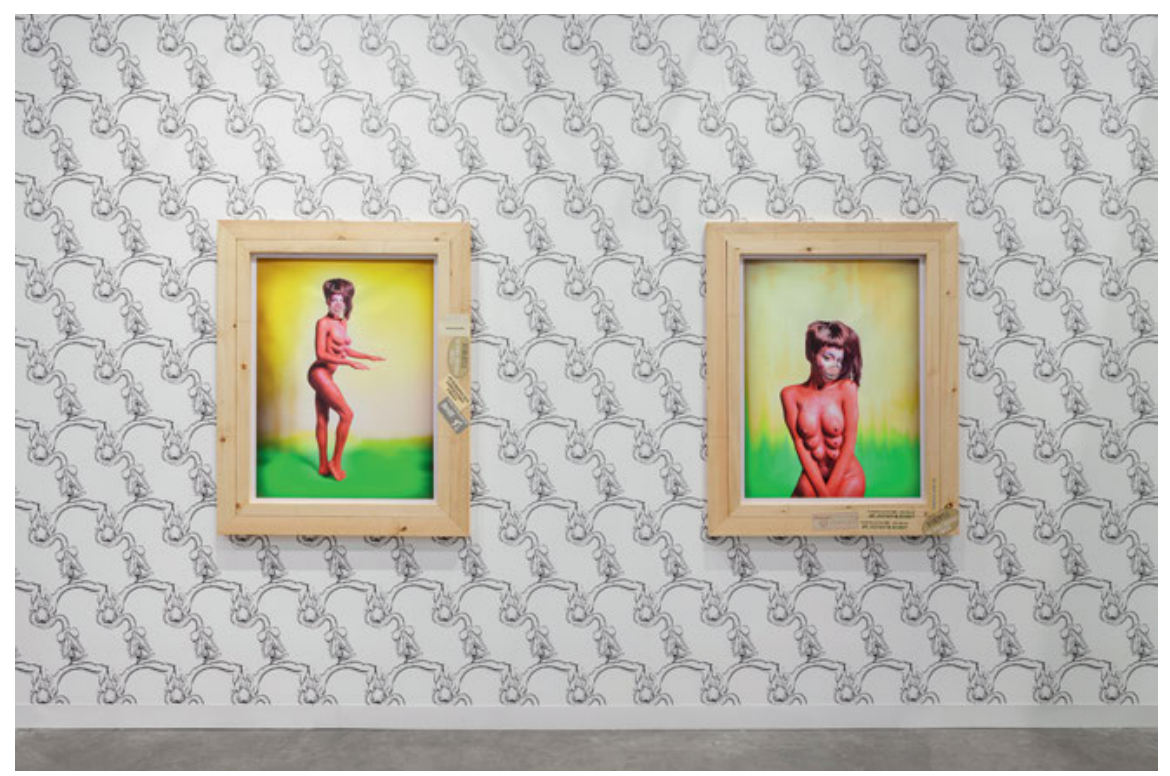

Abb. 2: Ausstellungsansicht, Project Native Informant / Juliana Huxtable an der Art Basel Hong Kong 2019, Courtesy of the artist and Project Native Informant, London.

Indem Huxtable für die Bilder ihrer Trans`Spezies-Wesen ihren eigenen Körper inszeniert, der trotz seiner Hybridisierung als humanoides Tierwesen bewusst geschlechtlich und ethnisch markiert bleibt, verschränkt sie mehrere Ebenen soziopolitischer Identitätskategorisierungen. Eines der beiden Bildmotive aus den insgesamt sechs digital manipulierten Fotografien in der Serie Zoosexuality zeigt Huxtable als Echsenfrau, die sich lasziv 
auf einem Bett räkelt und den Betrachtenden einen verführerischen Blick zuwirft (Abb. 3). Das kitschige Dekor des Zimmers und das gedimmte Licht, das von verschiedenen Lichtquellen her bunt schimmert, erwecken Assoziationen an ein Bordellzimmer oder - denn womöglich ist ein Bildschirm Quelle des diffusen grünlichen Lichts - an überstilisierte Räume von Webcam-Girls, die ihre sexuellen Dienste über das Internet anbieten. Das Mensch / Tier-Wesen ist nackt und trägt außer Schmuck nur ein Paar grüne High Heels, was den Fetischcharakter der Szenerie noch verstärkt. An Stelle von Huxtables Gesicht formt sich ein anthropomorphisierter Kopf einer Cartoon-Echse, unter dessen transluzider Oberfläche das Antlitz der Künstlerin noch schwach zu erkennen ist. Neben dieser digitalen Hybridisierung markiert Huxtable ihre Transformation zum Animalischen überdies auch über die Bemalung ihres Körpers in einer echsenähnlichen Musterung. Die nicht ganzflächig aufgetragene Körperbemalung lässt jedoch die Schwarze Hautfarbe Huxtables noch deutlich erkennen.

Die Identifikation Huxtables mit einem Reptil lässt sich vor dem Hintergrund der bewussten Zurschaustellung Schwarzer Hautfarbe als ikonographischer Hinweis auf den religiösen Mythos der Nuwaubian Nation lesen, den die Künstlerin auch in früheren Werken bereits zitiert (vgl. Schicharin 2017, $5 \mathrm{f}$.); sie begründet dies damit, dass das alternative Glaubensmodell der Sekte des Nuwaubianismus der Überzeugung entstammt, dass Schwarze Menschen direkte Nachkommen von reptilen Aliens seien, weiße Menschen hingegen von diesen ursprünglichen göttlichen Kreaturen erst erschaffen wurden und später die Weltherrschaft an sich gerissen haben. ${ }^{7}$ Mit der Anlehnung an nuwaubianische Echsenfiguren beruft sich Huxtable auf einen spekulativen Narrationsmodus von blackness, der sich unter den Begriff »Afrofuturismus« subsumieren lässt.

Der Vorschlag, bestimmte Formen Schwarzer Kulturproduktion ${ }^{8}$ seit den 1960er Jahren mit dem Begriff »Afrofuturismus» zu bezeichnen, geht

7 Huxtable spricht über ihre Faszination mit dem Kult in diversen Interviews. Siehe u.a. Hurr (2014). Huxtable ist selbst keine Angehörige dieser Sekte. Andere Werke Huxtables, die auf Nuwaubianismus referieren sind z. B. Nuwaubian Princess (2013) oder Untitled in the Rage (Nibiru Cataclysm) (2015). Vgl. dazu auch Krause-Wahl (2018), 579-581.

8 Das Spektrum an afrofuturistischen Disziplinen ist äußerst vielfältig und beinhaltet unter anderem Literatur, visuelle Kunst, Musik, Film und Mode. Die Revision des Afrofuturismus des 20. Jahrhunderts durch Reynaldo Anderson und Charles E. Jones im Jahr 2016 zählt neu auch die Dimensionen der Metaphysik, Ästhetik, theoretische und angewandte Wissenschaft und Sozialwissenschaften dazu (Anderson/Jones 2016, x). Zu den bekanntesten Vertreter_innen des Afrofuturimus gehören u.a. der Musiker Sun Ra und Schriftsteller_innen wie Octavia Butler, Audre Lorde und Samuel R. Delany. 


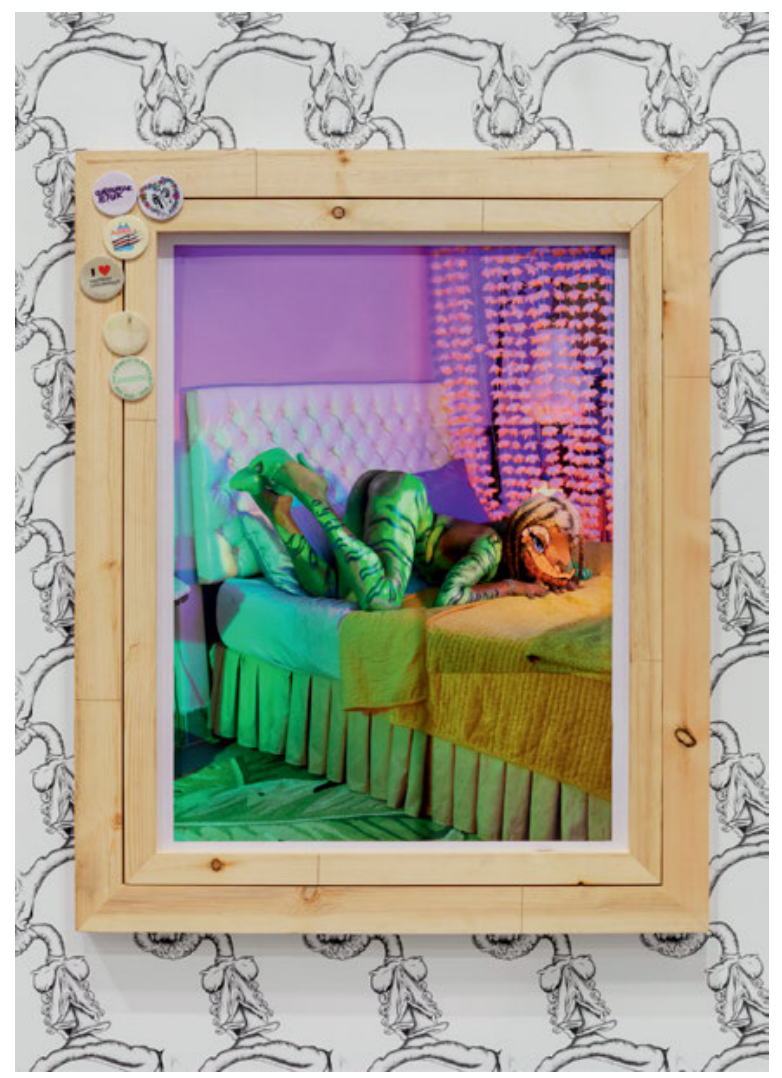

Abb. 3: Juliana Huxtable, Untitled, 2019, 12 colour archival ink auf Leinen, Collage, selbstgemachte Badges in Künstlerrahmen, $137 \times 107 \times 6 \mathrm{~cm}$, Courtesy of the artist and Project Native Informant, London.

auf einen 1994 veröffentlichten Aufsatz des amerikanischen Kulturkritikers Mark Dery mit dem Titel »Black to the Future« zurück. Als Afrofuturismus begreift Dery eine Bewegung Schwarzer Kunstschaffender, die sich mit Themen und (Diskriminierungs-)Erfahrungen der afrikanischen Diaspora auseinandersetzen, indem sie sie in spekulative Visionen einer Schwarzen, oftmals technologisierten Zukunft übersetzen (vgl. Dery 1994, 180). Spekulative Fiktion, so fasst Ramon Amaro $(2018,17)$ unter Bezug auf Dery zusammen, "offers the black body a method by which the alienness of terrestrial belonging is re-scripted, re-coded and re-organized into alternative narratives of being and becoming [...].« Grundsätzlich zeigen afrofuturistische künstlerische Narrative auf, dass Identität auf der Basis von Hautfarbe ein Abstraktum ist, das keine fixierbare transzendentale oder körperliche Form hat, sondern performativ und erst in Relation zu Diskursen und Pra- 
xen der Rassifizierung hervorgebracht wird. Mit dem Aufrufen des Topos des Aliens, beziehungsweise generell des Posthumanen, kritisieren Afrofuturist_innen einerseits die Exklusion Schwarzer Subjekte von der politischen Anatomie des "Menschen", wie sie in der Theorie des westlichen Humanismus dargestellt wird und auf deren Grundlage diskriminierende Praxen legitimiert werden (vgl. Eshun 2003, 298 f.; Samatar 2017, 181 f.). 9 Andererseits eröffnet die Identifikation mit dem Außerirdischen die Möglichkeit, Schwarze Erfahrungen und Identitäten abseits normativer und unterdrückender Strukturen zu denken (Samatar 2017, 181).

Der Begriff des Afrofuturismus, der bei Dery (1994) ein Projekt der Imagination einer Zukunft frei von Kolonialismus und rassistischer systemischer Gewalt bezeichnete, wurde parallel zu der wachsenden afrofuturistischen Kunstproduktion in den vergangenen Jahren von Forschenden auf vielfältige Weise diskutiert und erweitert. Der Bezug auf die Geschichte der Rassifizierung dient aktuellen afrofuturistischen Projekten oftmals nicht mehr dem Zweck einer utopischen Revision und Reappropriation einer "verlorenen" Vergangenheit (und Zukunft) Schwarzer Subjekte. Anstatt "Schwarzsein" von historischen Mechanismen der Rassifizierung lösen zu wollen, geht es laut der Kunsthistorikerin Tiffany Barber neueren Afrofuturismen um eine kritische Auseinandersetzung mit diesen unterdrückenden Signifizierungsprozessen:

$[W]$ here instantiations of post-blackness seek to disrupt a relation between blackness and a history of racialization, [...] Afrofuturism holds onto this history of racialization and troubles it by working within it. (Barber 2016, 11)

Mit dem visuellen Bezug auf die alternative Erzählung der Nuwaubian Nation durch die Identifikation mit dem echsenartigen Alien spielt Huxtable direkt auf die Geschichte weißer Herrschaft über Schwarze Menschen an. Sie projiziert die Geschichte der Sklaverei in ein spekulatives Zukunftsszenario, um sie mit gegenwärtigen Mechanismen systemischer Gewalt zu vergleichen.

\section{trans*: Intersektionale Figuren zwischen Fiktion und Realität}

Huxtables deutlich sexualisierte Inszenierung des Echsenmenschen formuliert über die Thematisierung der Rassifizierung hinaus eine weitere Kritik an normativen Konzeptionen von Identitäten und Körpern. In den

9 Vgl. zum Verhältnis von Rassismus und (Post-)Humanismus auch Weheliye, 2002. 
beiden anderen in der Ausstellung Zoosexuality gezeigten Varianten dieses Echsen-Bildmotivs lässt sich erkennen, dass die Brüste Huxtables nicht wie der größte Teil des restlichen Körpers in einem Reptilienhaut-Motiv bemalt wurden. Es scheint, als würde die Künstlerin bewusst den Blick auf die weibliche Brust lenken wollen, um zu verdeutlichen, dass der Figur nebst der rassifizierten auch eine vergeschlechtlichte minoritäre Position zukommt. Die zweifach sentblößte Brust der Figur - nackt und unbemalt und dergestalt sowohl weiblich als auch ethnisch markiert - stellt visuell eine Verbindung zwischen verschiedenen Formen der Unterdrückung her. Sexualisierung und Rassifizierung kombinieren sich auf dem sekundären Geschlechtsmerkmal dieses nicht ganz menschlichen Wesens, das sich lasziv und unterwürfig den Blicken der Betrachtenden hingibt. Das bordellhaft anmutende Interieur weckt, den Aspekt der Sexualisierung noch verstärkend, Assoziationen an Prostitution, die - sofern sie Zwangsarbeit ist - als moderne Form der Versklavung betrachtet werden kann (vgl. Bales 2007; Deckha 2019, 195).

Gerade vor der Linse des Topos Sexarbeit ist es von Bedeutung, dass über Huxtables fotografische Selbstinszenierungen als Hybridwesen hinaus - die Botschaften an den Bildrahmen der Werke in Zoosexuality die Art der dargestellten vergeschlechtlichten Verkörperung nochmals spezifizieren: "GENETIC LIBERATIONISTS FOR TRANS-SPECIES RIGHTS « lautet die Forderung auf einem den Bildrahmen schmückenden Pins, andernorts lässt sich das Symbol einer Hundepfote in den Farben der Transgender-Flagge erkennen. Mit diesen an gängige Botschaften der Protestkultur erinnernden Signifikanten überspitzt Huxtable identitätspolitische Debatten um die Rechte transgeschlechtlicher Subjekte und gibt den Hinweis darauf, dass die Wesen als doppelt trans ${ }^{\star}$ - trans`spezies und trans ${ }^{\star}$ gender - gelesen werden sollen. Das Prostitutionsszenario in Huxtables Bildern spielt womöglich auf die Tatsache an, dass Schwarze transgeschlechtliche Frauen aufgrund ihrer marginalisierten Position stark von strukturellen Ungleichheiten betroffen sind und oft keine andere Wahl haben, als mit Sexarbeit ihren Lebensunterhalt zu verdienen (vgl. Wilton / Short 2016, 148 f.). Rodrick A. Ferguson verdeutlicht in seinem Buch "Aberrations in Black: Toward a Queer of Color Critique«, dass race, Geschlecht, Sexualität und Klasse immer in einem wechselwirkenden Verhältnis zueinander stehen und keine voneinander zu trennenden Kategorien sind (Ferguson 2004). Die Verkörperung von Transgeschlechtlichkeit und Schwarzsein bezeichnet in diesem Gefüge eine ganz spezifische Intersektion, denn, so verdeutlicht Amber Johnson: "The Black trans body is more than a Black body, it is more than a gendered body, it is more than a 
trans body. It is a body that meets at specific, fluid, always changing intersections." (Johnson 2020, 21).

Das sexualisiert inszenierte Echsenwesen ist eine intersektionale Figur, die gleich mehrere Ebenen gesellschaftlicher Diskriminierung von Minoritäten verkörpert. Indem Huxtable einen Schwarzen transgeschlechtlichen Körper ins Zentrum ihrer afrofuturistischen Vision stellt, öffnet sie den Raum, um verschiedene hegemoniale Narrative miteinander in Verbindung zu bringen und gleichzeitig zu unterminieren. Dass die Ausstellung Zoosexuality sowohl rassistische als auch explizit heteronormative Strukturen und Asymmetrien hinterfragt, lässt sich auch an der ornamentalen Struktur der Tapete, vor deren Hintergrund die gerahmten Werke aufgehängt sind, ablesen. Ihr Motiv - ein Netzwerk, welches menschliche Geschlechtsorgane mit Oktopustentakeln, Verdauungstrakten, Pilzen und anderen organischen Formen verwuchert - lässt die Binarität von Geschlechtern und Geschlechtskörpern an den Gattungsgrenzen des Menschlichen »explodieren«: Dichotomien zwischen männlich / weiblich, aber eben auch zwischen Mensch/Tier und Mensch/Umwelt kollabieren, werden ineinander verwoben und sind nicht mehr voneinander zu trennen. Es zeigt sich hier das Konzept einer queeren Wesensform, die unbegrenzt, interrelational und multi-geschlechtlich ist, und welche sich in einem infiniten Prozess des Werdens durch permanente Interaktion mit multiplen Anderen befindet.

Die Tapete, die sich als Referenzrahmen für die Selbstporträts Huxtables lesen lässt, untermauert, dass mit den Darstellungen menschlichanimalischer Hybridwesen Topoi der Geschlechtlichkeit und Sexualität, wie auch der Interdependenz zwischen verschiedenen Spezies verhandelt werden. Huxtable spielt mit instabilen Signifikanten, die ihre Bilder zwischen Utopie, Dystopie und Realität fluktuieren lassen. Die nicht-hierarchische und unauflösbare Verwobenheit verschiedener minoritärer und dominanter Subjektpositionen im ornamentalen Muster der Tapete lässt die Interpretation $\mathrm{zu}$, dass hier womöglich das Bild einer utopischen $\mathrm{Ge}-$ sellschaftsstruktur abseits heteronormativer und speziesistischer Machthierarchien gezeichnet wird. Von Weitem betrachtet erweckt die geordnete geometrische Struktur der Tapete jedoch die Assoziation an einen Stacheldrahtzaun ${ }^{10}$, womit die prekären Lebensbedingungen von Schwarzen Menschen, und besonders von Schwarzen transgeschlechtlichen Menschen, wieder zurück ins Bild gerückt werden. Der Stacheldraht verweist einerseits, so könnte man argumentieren, auf ein Gefangensein im 
Netzwerk heteronormativer Identitätskonstruktionen, aus dem nur binäre Geschlechtsidentität als »lebbare« Option hervorgebracht wird. ${ }^{11}$ Das Abweichen von diesem binären System hat zur Folge, dass nicht-binäre und transgeschlechtliche Menschen gesellschaftlich auf den Status des Animalischen bzw. des Subhumanen reduziert werden. Auch rassifizierte Subjekte sind, worauf ich später noch zurückkommen werde, Opfer solcher gewaltvollen Zuschreibungen. Gerade im amerikanischen Kontext liest sich der Stacheldrahtzaun andererseits aber auch als Symbol für den industriellen Gefängniskomplex der USA, dessen politische Ökonomie, wie viele kritische Stimmen argumentieren, auf einem rassistisch voreingenommenen Justizsystem beruht. ${ }^{12}$ Freiheitsberaubung und Zwangsarbeit im kapitalistischen amerikanischen Gefängnissystem zeugen davon, dass Sklaverei auch heute noch unter einem neuen, legalen Deckmantel praktiziert wird (vgl. u. a. Bales 2007; Deckha 2019; Nocella 2019). Indem Huxtable mit dem Motiv der Tapete in binären Strukturen gefangene Geschlechtlichkeit mit den Sphären von Gefängnis und Sklaverei verbindet, scheint sie auch das Augenmerk darauf richten $\mathrm{zu}$ wollen, dass Schwarze Trans`Frauen aufgrund ihrer mehrfachen sozioökonomischen Benachteiligungen besonders gefährdet sind, inhaftiert zu werden. Gerade weil Sexarbeit oft die einzige Einkommensquelle für Schwarze Trans`Frauen in den USA darstellt, fallen sie häufiger den gewaltvollen Mechanismen der Rassifizierung durch das Justizsystem zum Opfer:

Many of the trans people who are incarcerated are funneled through the sexwork-prison-release-repeat pipeline wherein released former prisoners cannot not find stable employment due to their trans-identity and their record and are forced back into the high risk area of sex-work. The bodies of transwomen are often exoticized, othered, objectified, commodified, and thus employed in a project of racialization through the mechanisms of sex work and prison. (Bevensee 2014, 103).

Huxtables afrofuturistische Fiktion bewegt sich, so behaupte ich, im Raum zwischen gelebter Realität und spekulativer Fantasie, um die spezifischen systemischen Gewaltmechanismen, durch welche Schwarze Trans`Frauen

11 Juliana Huxtable selbst ist intersexuell geboren und wurde bei Geburt dem männlichen Geschlecht zugewiesen. Da die Künstlerin in den besprochenen Werken sonst keine konkreten Hinweise auf den Topos der Intersexualität zu machen scheint, gehe ich in dieser Arbeit nicht weiter darauf ein.

12 Rund 60\% der Inhaftierten in US-amerikanischen Gefängnissen sind people of color, während sie gleichzeitig nur 30\% der amerikanischen Gesamtbevölkerung ausmachen. Vgl. Nocella 2019, 205. 
unverhältnismäßig benachteiligt werden, sichtbar zu machen. Huxtables Strategie der Sichtbarmachung queerer Schwarzer Subjekte, lässt sich mit Amandine Faucheux (2017) damit als "queerer Afrofuturismus" benennen. Unter den Begriff des "Queer Afrofuturism« fallen, so Faucheux, diejenigen afrofuturistische Projekte, "in which race is inextricably tied to gender and sexuality in such a way that it is impossible to talk about one without always already signifying the other." (Ebd., 565). Dem queeren Afrofuturismus liegt ein subversives Potential zugrunde - einerseits, weil er die Konstruktion von Identität über visuelle Marker von »Otherness" kritisch hinterfragt, und andererseits "by reclaiming the positions of black and/or queer subjects in historical narratives where they have been violently erased." (Ebd., 567). Huxtables "queering" des Afrofuturismus lässt sich so auch als "Werkzeug des Widerstands" (vgl. Stotter 2016) verstehen - oder als "Great Refusal", wie es Queer of Color Theoretiker José Esteban Muñoz beschreiben würde - in welchem das Potential angelegt ist, neue Denkmodi zu generieren, »because art manifest [sic] itself in such a way that the political imagination can spark new ways of perceiving and acting on a reality that is itself potentially changeable.« (Muñoz 2009, 135).

\section{Nutztiere als Sexarbeiterinnen: Verknüpfungen von institutionalisierter speziesistischer, rassistischer und sexistischer Gewalt}

Das queere Projekt der Sichtbarmachung rassistischer und sexistischer Diskriminierungsnarrative erweitert sich mit dem Blick auf das zweite humanoide Tierwesen in der Werkserie Zoosexuality um eine dritte Dimension der Kritik, die ebenfalls im Motiv des Stacheldrahtzauns bereits Resonanz findet. Auch Huxtables Selbstinszenierungen als Hybridin zwischen Mensch und Schwein verhandeln Thematiken (sexueller) Ausbeutung. Allerdings thematisiert Huxtable hier noch eine weitere Ausformung der Abwertung von minoritären Subjekten: diejenige der industriellen Ausbeutung von Nutztieren. Das entweder in unterwürfiger Pose auf einen Melkstuhl aufgestützte, oder auffordernd lasziv in die Kamera blickende Frau $^{\star} /$ Schwein-Hybridwesen (Abb. $2 \& 4$ ) ist überdeutlich geschlechtlich markiert. Die Schweinezitzen sind - wenngleich hypertroph überformt und dreifach multipliziert - als menschlich-weibliche Brüste zu erkennen. Die Bilder Huxtables verknüpfen spielerisch die Hierarchisierung von Spezies, deren prominenteste und gewaltvollste Ausformung die industrielle Nutztierhaltung ist, mit patriarchalen Machtmechanismen von Sexua- 


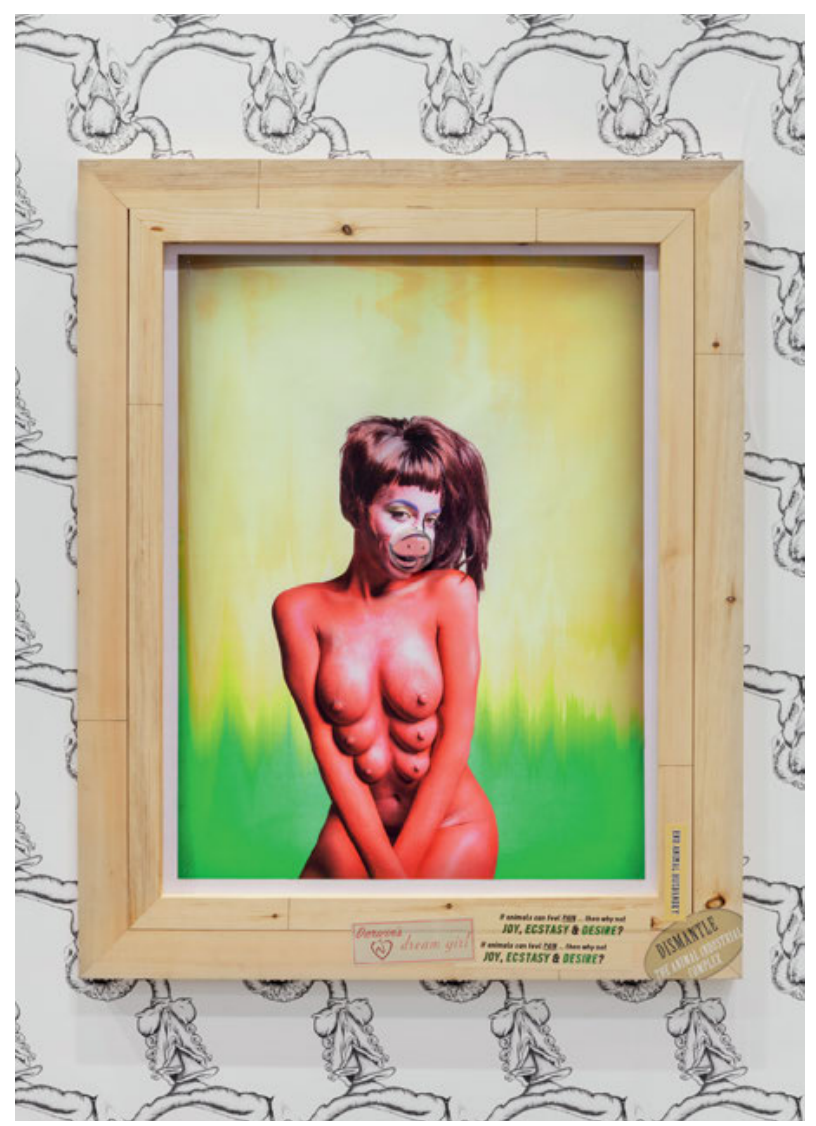

Abb. 4: Juliana Huxtable, Untitled, 2019, 12 colour archival ink auf Leinen, Collage, selbstgemachte Badges in Künstlerrahmen, $137 \times 107 \times 6 \mathrm{~cm}$, Courtesy of the artist and Project Native Informant, London.

lisierung und Objektivierung vergeschlechtlichter Subjekte. Während das neckische Lächeln der Hybridin eine Einladung an die Betrachtenden auszusprechen scheint, den Blick auf ihre offen präsentierten Brüste zu richten, und auch ein Aufkleber am unteren Rand des Bildrahmens vermeintlich implizieren möchte, dass das erotische Begehren auf Gegenseitigkeit beruht - »If animals can feel PAIN ... then why not JOY, ECSTASY \& DESIRE? - , senden die Sticker rechts daneben dazu deutlich im Widerspruch stehende politische Botschaften: "End Animal Husbandry" ("Setzt ein Ende der Viehzucht») oder "Dismantle the Animal Industrial Complex" (etwa: "Reißt den Tierindustriekomplex nieder«). Die Forderung, der industriellen Ausbeutung von Tieren ein Ende zu setzen, trifft auf die Einladung, Tiere als Wesen zu sehen, an denen sexuelle Handlungen voll- 
zogen werden dürfen. Die Bildstrategie Huxtables scheint darin zu bestehen, den male gaze $e^{13}$ ad absurdum zu führen, um einen Erkenntnisprozess $\mathrm{zu}$ generieren: Einerseits werden die Betrachtenden über Bildkomposition und Inszenierung dazu geführt, eine Verbindung zwischen der Ausbeutung von Tieren und der Sexualisierung von Frauen ${ }^{\star}$ herzustellen und diese kritisch zu reflektieren; gleichzeitig werden sie geleitet, gedanklich gesellschaftliche Sexualisierungsprozesse auf nicht-menschliche Tiere zu übertragen. In einer scheinbar widersprüchlichen Bewegung wird die Enttabuisierung des zoosexuellen Begehrens gefordert und gleichzeitig das männliche heterosexuelle Begehren kritisiert: Warum ist die Sexualisierung von Weiblichkeit weit verbreitet während Zoosexualität verpönt bleibt? Sind sie nicht sogar vergleichbar? Wie kann man legitimieren, dass das eine verboten sein soll, während das andere tagtäglich praktiziert wird?

Verbindungen von Sexualisierung und Speziesierung waren auch Thema in Huxtables Ausstellung Interfertility Industrial Complex: Snatch the Calf Back, in deren Zentrum die Darstellung einer Interspezies-Milchkuh stand, die - Hände hinter dem Rücken zugeschnürt und vornüber gebeugt - gewaltvoll von einer Melkmaschine gemolken wird (Abb. 5). Die hypersexualisierte Frau/Kuh-Figur wirft die Frage nach sexueller Einvernehmlichkeit auf und weitet sie auf das Gebiet der Nutztierindustrie aus, in welcher repetierte künstliche Insemination und Reproduktion feste Bestandteile des industriellen Prozesses sind. ${ }^{14}$ Ein in der Ausstellung gezeigtes Video, auf dem nur die in violettem Kuhmuster bemalte Mundpartie Huxtables zu sehen ist, erzählt die Geschichte einer auf einem Bauernhof aufgewachsenen Frau ${ }^{\star}$ und ihrer (erotisch motivierten) Sehnsucht danach, eine Milchkuh zu sein. Die Erzählung ihrer unterwürfigen sexuellen Fantasie, in welcher sie vom Bauern gemolken wird, endet mit einer unerwarteten Wendung: "I wanted the burning desire of the farmer, my sex in service of his empire ... To have my swollen teats milked. There was just one irresolvable plot complication: I felt like I should be paid for my labor. $\aleph^{15}$ Mit der Forderung nach Bezahlung für die Sexarbeit der menschlichen Milchkuh wird die Kategorie "Arbeit" von der Sphäre der menschlichen Gesellschaft auf die Sphäre der Nutztiere übertragen, scheinbar um zu zeigen, dass auch nichtmenschliche Lebewesen in Diskurse systemischer Gewalt mit eingeschlossen werden sollen.

13 Zur feministischen Geschichte des male gaze in der Kunst vgl. u.a.: Mulvey [1975] 2010; Olin 2003; Kravagna 1997.

14 Vgl. dazu auch die Ausstellungsrezension von Shama Nimkar (2019).

15 Link zum Video: https://contemporaryartdaily.com/2019/10/juliana-huxtable-atreena-spaulings-2/ (25.02.2020). 


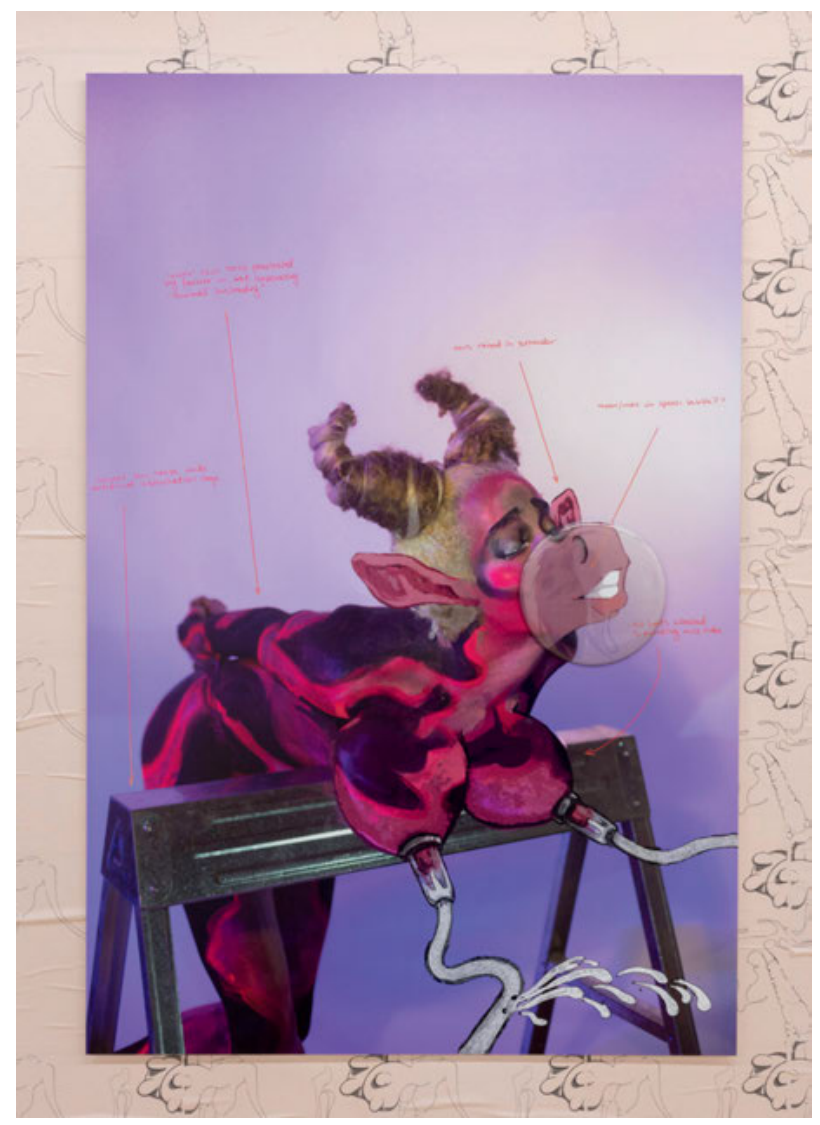

Abb. 5: Juliana Huxtable, COW 3, 2019, Inkjet befestigt auf Dibond, $100.96 \times 67.31 \mathrm{~cm}$, Courtesy of the artist and Reena Spaulings Fine Art, NY/LA.

Dass hierarchische Mensch/Tier-Beziehungen immer schon verwickelt sind mit Formen der Unterdrückung wie Sexismus und Rassismus, argumentieren Theoretiker_innen der intersektionalen »Critical Animal Studies", wie beispielsweise Erika Cudworth $(2011 ; 2015)$ und Maneesha Deckha (2019), ebenso wie Kritiker_innen/Aktivist_innen wie Aph Ko (2019) oder A. Breeze Harper (2010), die sich an der Intersektion von "Critical Race Theory" und dem Feld des sogenannten "Black Veganism" ansiedeln lassen. Konzeptionen von Mensch / Menschsein und Tier/Tiersein, so sind sich die genannten Denker_innen einig, gehen immer schon von einer politischen Anatomie aus, die auf normativen sexualisierten, rassifizierten, eurozentrischen und anthropozentrischen Idealen beruht. Alle von diesen Normvorstellungen abweichenden Körper und Subjektivitäten haben in diesem Sinne nie ganz den Status des Mensch-Seins 
erlangt und fallen diskriminierenden und exkludierenden Prozessen des "Othering " zum Opfer. "Anders menschliche« Menschen ("otherwise human", Braidotti 2014) - Frauen, LGBTQ+, People of Color und Menschen mit Behinderung - wurden politisch und sozial vermehrt mit denjenigen, die »anders als Menschen" (»other-than-human«, Braidotti 2014) sind, gleichgesetzt. Genauso wie sich die Legitimation industrieller Viehzucht über den Status von Tieren als subhumane Wesen ergibt, sind es auch Mechanismen der Dehumanisierung, die der institutionalisierten Gewalt an Schwarzen und transgeschlechtlichen Menschen zugrunde liegen (vgl. Deckha 2019, 189 f.; Butler 2018, 110 f.).

Erika Cudworths Aussage »Animal labor within capitalism is slave labor" aus dem Aufsatz "Intersectionality, Species and Social Domination" könnte genauso gut ein Protest-Slogan aus einer der besprochenen Ausstellungen von Juliana Huxtable sein. Visuell wie auch sprachlich - der Slogan "DISMANTLE THE ANIMAL INDUSTRIAL COMPLEX" ist eine Anspielung auf den stehenden Begriff des "Prison Industrial Complex" in Amerika - führt Huxtable die enge Verknüfpung rassistischer, sexistischer ${ }^{16}$ und speziesistischer Unterdrückungsformen vor; so macht sie nicht nur deutlich, dass sie alle in einem komplexen Herrschaftssystem miteinander verwickelt sind, sondern dass man die eine ohne die anderen nicht eliminieren kann (vgl. dazu auch Nocella 2019, 205).

\section{Huxtables Kunst als (Re)Konfigurationen der Welt}

In Juliana Huxtables futuristischen Bildern von Trans`spezies-Wesen überlagern sich gleich mehrere Kritiken an gesellschaftlichen Ungleichheitsverhältnissen: Erstens greifen sie den in unserer Gesellschaft tief verankerten Speziesismus an, also die Diskriminierung und Hierarchisierung von Lebewesen aufgrund ihrer Zugehörigkeit zu einer Spezies; zweitens kritisieren sie Prozesse der Speziesierung und Dehumanisierung minoritärer menschlicher Subjekte aufgrund von Rassifizierung, und drittens auf der Basis von Geschlechternormen. Die futuristischen Hybridwesen von Juliana Huxtable erweisen sich durch das geschickte Zusammenspiel komplexer visueller Signifizierungsprozesse so gleich mehrfach als trans` und schöpfen damit, analog zu Eva Hayward in ihrem Aufsatz »More Lessons

16 Dass die Nutztierindustrie, wie Erika Cudworth argumentiert, patriarchal aufgebaut ist, ist eine weitere Bedeutungsebene, die sich auch in Huxtables Werken wiederzufinden scheint. Nutztiere sind größtenteils weiblich und werden strategisch zu Zwecken der Profitmaximierung gezüchtet und ausgebeutet (Cudworth 2015, 100). 
from a Starfish" (Hayward 2008), das kritische Potential eines Zusammendenkens von "transspezies" und "transgender" aus (vgl. Wark 2020). Die beiden Ausstellungen Huxtables aus dem Jahr 2019 sind informierte und intellektuelle Gesamtkunstwerke, in denen rassistische, sexistische und speziesistische Narrative aus der Vergangenheit und der Gegenwart visualisiert und in ein spekulatives Zukunftsszenario transportiert werden. Durch diesen Futurismus in Kombination mit den komplexen Bezügen auf zeitgenössische theoretische Debatten um verschiedene Formen systemischer Gewalt lässt sich Huxtables Kunst als Beitrag zu einem zeitgenössischen Afrofuturismus verstehen. Denn - so behauptet Ytasha Womack, eine der prominentesten aktuellen Stimmen des Afrofuturismus - Afrofuturismus ist immer beides: Eine martistic aesthetic and a framework for critical theory" (Womack 2013, 9).

Huxtables Hybridwesen lassen sich begreifen »als performative und generative Entitäten, die aktiv an der Re(kon)figurierung der Welt teilhaben.« (Liebsch 2019, 272). Sie sind nicht nur kritische Kommentare, sondern auch künstlerische Interventionen in systemische Ungleichheitsverhältnisse. Ihre oftmals instabilen Symbole leiten die Betrachtenden durch eine Kette an widersprüchlichen Denkbewegungen hindurch und sind so der Zündstoff für transformative Erkenntnisprozesse. Es scheint der Künstlerin nicht darum zu gehen, den moralischen Zeigefinger zu heben, indem sie zeitgenössische (identitäts)politische Konflikte einfach hyperbolisch vergrößert und sie in eine dystopische Zukunft transportiert, die uns lehrmeisterhaft zeigen soll, was passieren wird, wenn wir an unseren Handlungs- und Denkweisen nichts ändern. In der Unentschiedenheit, die den posthumanen Wesen Huxtables eigen ist - verspürt das Kuhwesen (Abb. 5) denn nun Lust beim Melken, oder verzieht sie ihr Gesicht doch eher vor Schmerz? - liegt die Kraft, festgefahrene Gesellschaftsstrukturen außerhalb der immer gleichen Diskurse neu zu hinterfragen.

\section{Literatur}

Amaro, Ramon (2018): Afrofuturism. In: Braidotti, Rosi / Maria Hlavajova (Hrsg.). Posthuman Glossary. London, 17-20.

Anderson, Reynaldo/Jones, Charles E. (2016): Introduction: The Rise of Astro-Blackness. In: Dies. (Hrsg.). Afrofuturism 2.0: The Rise of Astro-Blackness. Lanham, viixviii.

Bales, Kevin (2007): Ending Slavery: How We Free Today’s Slaves. Berkeley. 
Barber, Tiffany (2016): Cyborg Grammar? Reading Wangechi Mutu's Non je ne regrette rien through Kindred. In: Anderson, Reynaldo/Jones, Charles E (Hrsg.). Afrofuturism 2.0: The Rise of Astro-Blackness. Lanham, 3-25.

Bevensee, Emmi (2014): Transwomen, the Prison-Industrial Complex, and Human Rights. Neoliberalism and Trans-Resistance. In: Societies Without Borders 9 (1), 96-110.

Braidotti, Rosi (2014): Metamorphic Others and Nomadic Subjects. Text written for the exhibition by Oliver Laric at ar/ge kunst Galerie Museum, Bolzano and at Tanya Leighton, Berlin. https://artmap.com/tanyaleighton/exhibition/oliver-laric-2014 (12.08.2020).

Busta, Caroline (2019): Juliana Huxtable on Zoosexuality, Furries, and the Fetishization of Outrage [Interview]. In: Art Basel Stories (13.02.2019). https://www.artbasel.com/ stories/juliana-huxtable-project-native-informant-art-basel-hong-kong (14.07.2020).

Butler, Philip (2018): Making Enhancement Equitable: A Racial Analysis of the Term 'Human Animak and the Inclusion of Black Bodies in Human Enhancement. In: Journal of Posthuman Studies 1:2, 106-121.

Cudworth, Erika (2015): Intersectionality, Species and Social Domination. In: Nocella, Anthony J. / White, Richard J. / Cudworth, Erika (Hrsg.): Anarchism and Animal Liberation. Essays on Complementary Elements of Total Liberation. Jefferson, 93-107.

Cudworth, Erika (2011): Social Lives with Other Animals. Tales of Sex, Death and Love. Basingstoke.

Deckha, Maneesha (2019): The Subhuman as a Cultural Agent of Violence. In: Nocella, Anthony J. / George, Amber E. (Hrsg.). Intersectionality of Critical Animal Studies: A Historical Collection. New York, 187-202.

Dery, Mark (1994): Black to the Future: Interviews with Samuel R. Delany, Greg Tate, and Tricia Rose. In: Ders. (Hrsg.), Flame Wars: The Discourse of Cyberculture. Durham, 179-222.

Eshun, Kodwo (2003): Further Considerations on Afrofuturism. In: The New Centennial Review 3:2, 287-302.

Faucheux, Amandine (2017): Race and Sexuality in Nalo Hopkinson's Oeuvre; or, Queer Afrofuturism. In: Science Fiction Studies 44:3, 563-580.

Ferguson, Rodrick A. (2004): Aberrations in Black: Toward a Queer of Color Critique. Minneapolis.

Harper, Breeze A. (2010): Sistah Vegan: Black Female Vegans Speak on Food, Identity, Health, and Society. New York.

Hayward, Eva (2008): More Lessons from a Starfish: Prefixial Flesh and Transspeciated Selves. In: Women's Studies Quarterly 36:3\&4, 64-85.

Hurr, Hanna (2014): Juliana Huxtable (Interview). In: Mask Magazine 8 (September 2014). http://www.maskmagazine.com/the-street-issue/work/juliana-huxtable (12.08.2020).

Johnson, Amber (2020): Trans Identity as Embodied Afrofuturism. In: Luckett, Sharrell D. (Hrsg.). African American Arts: Activism, Aesthetics, and Futurity. Lewisburg, 15-28.

Klumbyte, Goda (2018): Trans`. In: Braidotti, Rosi / Maria Hlavajova (Hrsg.). Posthuman Glossary. London, 433-435.

Ko, Aph (2019): Racism as Zoological Witchcraft: A Guide for Getting Out. New York.

Krause-Wahl, Antje (2018): Ökonomien der sozialen Medien - Bildpolitik und Gemeinschaftsbildung in den digitalen Netzwerken der Gegenwartskunst. In: Zeitschrift für Kunstgeschichte 81 (2018), 575-587.

Kravagna, Christian (1997): Privileg Blick. Kritik der visuellen Kultur. Berlin. 
Liebsch, Katharina (2019): Zwischen Science Fantasy und ontologischer Politik. Künstlerischer Technomaterialismus jenseits der Geschlechtergrenzen. In: feministische Studien (2), 269-288.

Mulvey, Laura ([1975] 2010): Visual Pleasure and Narrative Cinema. In: Jones, Amelia (Hrsg.): Feminism and Visual Culture Reader. New York, 57-65.

Munder, Heike (Hrsg.) (2019): Producing Futures. A Book on Post-Cyber-Feminisms. Ausst.-Kat. Migros Museum für Gegenwartskunst. Zürich.

Muñoz, José Esteban (2009): Cruising Utopia: The Then and There of Queer Futurity. New York.

Nimkar, Shama (2019): Trans Artist Juliana Huxtable's Powerful Comparison Between Female Sexuality and Livestock Farming. In: Medium (11.12.2019). https://medium. com/@shamanimkar/trans-artist-juliana-huxtables-powerful-comparison-betweenfemale-sexuality-and-livestock-farming-7bfd05f0eed8 (12.08.2020).

Nocella, Anthony J. (2019): Animal Advocates for Prison and Slave Abolition. In: Nocella, Anthony J. / George, Amber E. (Hrsg.). Intersectionality of Critical Animal Studies: A Historical Collection. New York, 203-209.

Olin, Margaret (2003): Gaze. In: Nelson, Robert S./Shiff, Richard (Hrsg.): Critical Terms for Art History. Chicago; London, 318-329.

Reysen, Stephen / Plante Courtney N. / Roberts, Sharon E. / Gerbas, Kathleen C. (2020): My Animal Self: The Importance of Preserving Fantasy-Themed Identity Uniqueness. In: Identity 20:1, 1-8.

Samatar, Sofia (2017): Toward a Planetary History of Afrofuturism. In: Research in African Literatures 48:4 (Winter 2017), 175-191.

Schicharin, Luc (2017): Le corps abstrait d'une princesse nuwaubienne. L'extra terrestre comme figure de l'altérité dans l'oeuvre de Juliana Huxtable. In: Images Re-vues 14, 704-750.

Stotter, Magdalena (2016): Did you say Afrofuturism? On labelling art. In: Wikis der Freien Universität Berlin. https://wikis.fu-berlin.de/pages/viewpage.action?pageId= 646989083 (10.07.2020).

Wark, McKenzie (2020): Reality Cabaret: On Juliana Huxtable. In: e-flux journal 107. https://www.e-flux.com/journal/107/322326/reality-cabaret-on-juliana-huxtable/ (10.07.2020).

Weheliye, Alexander G. (2002): »Feenin«: Posthuman Voices in Contemporary Black Popular Music. In: Social Text 71 (20:2), 21-47.

Wilton, Leo/Short, Ellen L. (2016): „Black Lives Matter«: Structural Violence, Agency, and Resiliency in Black Transgender Women's Communities. In: Dies.: Talking about Structural Inequalities in Everyday Life: New Politics of Race in Groups, Organizations, and Social Systems. Charlotte, 139-164.

Womack, Ytasha L. (2013): Afrofuturism: The World of Black Sci-Fi and Fantasy Culture. Chicago. 